\title{
ORIGINAL
}

\section{LESIONES POR ACCIDENTE DE TRABAJO EN ESPAÑA: COMPARACIÓN ENTRE LAS COMUNIDADES AUTÓNOMAS EN LOS AÑOS 1989, 1993 Y 2000}

Fernando G. Benavides (1), Emilio Castejón (2), Maria Teresa Giráldez (3), Nùria Catot (1) y Jordi Delclós (4)

(1) Unitat de Recerca en Salut Laboral. Universitat Pompeu Fabra. Barcelona

(2) Instituto Nacional de Seguridad e Higiene en el Trabajo. Barcelona

(3) Ministerio de Trabajo y Asuntos Sociales. Madrid

(4) Universidad de Texas. Houston

\section{RESUMEN}

Fundamento: A pesar de que en España la incidencia de lesiones por accidentes de trabajo (LAT) es una de las más elevadas de la Unión Europea, sido escasa. El objetivo de este estudio fue comparar la incidencia de LAT mortales y no mortales por comunidades autónomas (CCAA) en 1989,1993 y 2000.

Métodos: Los datos de las LAT se obtuvieron del Ministerio de Trabajo y Asuntos Sociales y los denominadores del Instituto Nacional de Estadística. La incidencia se ha calculado para LAT mortales y no mortales, ajustando separadamente por sexo, edad, actividad económica y tipo de contrato. Para cada CCAA se calculó el riesgo relativo (RR) ajustado por actividad económica y tipo de contrato, tomando como referencia la media española.

Resultados: Cataluña y Baleares presentaron los RR de LAT no mortales más elevados. Respecto a las LAT mortales, fueron Asturias y País Vasco donde el RR fue más alto. Madrid y Cataluña, por el contrario, presentó el RR más bajo en lesiones mortales.

Conclusiones: El exceso de riesgo de LAT encontrados en algunas CCAA puede ser explicado, una vez descartadas parcialmente las diferencias en las actividades económicas y el tipo de contrato, en términos bien de diferencias en la notificación de la LAT y/o de la población en riesgo, o bien, hipótesis más plausible, en términos de diferencias en las condiciones de trabajo de las empresas de estas CCAA. Es necesario seguir estudiando con más detalle estas diferencias.

Palabras clave: Traumatismos. Salud laboral. Prevención.

Correspondencia:

Fernando G. Benavides.

Unitat de Recerca en Salut Laboral. Departament de Ciències Experimentals i de la Salut. Universitat Pompeu Fabra.

C/ Doctor Aiguader, 80. 08003 Barcelona.

Fernando.Benavides@upf.es

Proyecto parcialmente financiado con una ayuda del Ministerio de Ciencia y Tecnología (BSA2001-0965) y de la Red de Centros y grupos de Epidemiología y Salud Pública.

\section{ABSTRACT}

Occupational Accident Injuries in Spain: A Comparison Among Autonomous Communities in 1989, 1993 and 2000

Background: Despite the incidence of occupational accident injuries in Spain being one of the highest in the European Union, this health problem has been research very little to date. This study was aimed at comparing the incidence of fatal and non-fatal occupational accident injuries by Autonomous Communities in 1989, 1993 and 2000.

Methods: The occupational accident injury data was obtained from the Ministry of Labor and Social Affairs, and the denominators from the Spanish National Institute of Statistics. The incidence was calculated for fatal and non-fatal occupational accident injuries, adjusting separately by sex, age, business activity and type of contract, taking the Spanish average as a reference.

Results: Catalunya and the Balearic Islands showed the highest relative risks of non-fatal occupational accident injuries. Regarding fatal occupational accident injuries, the Asturias and the Basque Autonomous Communities were the Communities where the relative risk was highest. Madrid and Catalunya, on the contrary, having showed the lowest RR for fatal injuries.

Conclusions: The excessive risk of occupational accident injuries found in some Autonomous Communities can be explained, after partially ruling out the differences in the business activities an type of contract, either in terms of differences in the reporting of the occupational accident injuries and/or of the population at risk, or rather, in terms of differences in the working conditions at the companies in these Autonomous communities, which is the most plausible hypothesis. These differences must be studied further in greater detail.

Key words: Trauma. Occupational health. Prevention. 


\section{INTRODUCCIÓN}

La incidencia de las lesiones por accidentes de trabajo en España es una de las más elevadas de los estados de la Unión Europea. Los datos de Eurostat muestran que la incidencia media española en el año 1999, último disponible, fue de 70,3 lesiones con baja laboral de más de tres días por 1.000 trabajadores cubiertos, frente a la media europea de 40,9 . Una diferencia que se mantiene en relación a la incidencia de lesiones mortales, que en España fue de 5,0 por 100.000 trabajadores cubiertos frente a los 2,9 de la media europea ${ }^{1}$.

El gasto ocasionado por estas lesiones en términos de asistencia sanitaria (atención médica y prestación farmacéutica), prestación económica (subsidio, pensiones, etc...) y gastos de administración ascendió a algo más de 4.357 millones de euros en el año 2000 , los cuales proceden de las cotizaciones que por esta cobertura pagan las empresas a la Seguridad Social, que en su mayor parte (un 95\% aproximadamente) gestionan las Mutuas de accidentes de trabajo y enfermedades profesionales ${ }^{2}$.

Entre las causas de las LAT se han señalado numerosos factores de riesgo ligados al puesto de trabajo (manipulación de cargas pesadas, herramientas y equipos de trabajo, turnos, antigüedad en el puesto, falta de control sobre la tarea, etc.) y al trabajador (edad, conductas, etc. $)^{3-5}$. En nuestro país, donde el estudio de este problema ha tenido un carácter principalmente descriptivo ${ }^{6}$, la investigación más analítica se ha centrado en el papel del tipo de contrato, habiéndose observado que los trabajadores con contrato temporal tiene entre dos y tres veces más riesgo de sufrir una LAT que los trabajadores con contrato indefinido ${ }^{7,8}$. Asimismo, se ha observado una relación temporal de la incidencia de LAT con el crecimiento económico ${ }^{9,10}$ y con la actividad económica de la empresa ${ }^{11}$, encontrándose grandes diferencias entre el riesgo de trabajar en la cons- trucción y, por ejemplo, en finanzas y ban$\mathrm{ca}^{12}$.

En cuanto a la prevención de este problema de salud la ley establece claramente que ésta es responsabilidad de las empresas ${ }^{13}$, las cuales deben disponer de los recursos preventivos necesarios para tratar de evitarlos (entre ellos una unidad básica sanitaria con la responsabilidad de la vigilancia de la salud), si bien las administraciones públicas, principalmente autonómicas, tiene un papel clave en el control y la promoción de estas actividades preventivas. A pesar de ello, como mostraba un reciente informe sobre riesgos laborales y su prevención ${ }^{14}$, cada día se producen unas 3 LAT mortales y unas 2.500 no mortales con baja en España.

Posiblemente, para que en nuestro país este problema comience a estar bajo control, una condición necesaria es que, como se ha señalado recientemente para las lesiones por accidentes de tráfico ${ }^{15}$, se reconozca plenamente como un problema sanitario.

El objetivo de este trabajo ha sido comparar la incidencia de LAT en los años 1989, 1993 y 2000 por Comunidades Autónomas (CCAA), teniendo en cuenta diversas variables de confusión, lo que no se había analizado hasta ahora, con el fin de describir patrones de distribución espacial y temporal que ayuden a identificar determinantes de este importante problema de salud que puedan ser útiles para su prevención.

\section{POBLACIÓN Y MÉTODOS}

Desde 1988 existe en España un sistema de información sobre LAT $^{16}$, que permite disponer de la información anual de los accidentes de trabajo ocurridos según, entre otras variables, el sexo, la edad, el tipo de contrato, la actividad económica de la empresa y la provincia donde está registrado el centro de trabajo. La edad del trabajador en el momento del accidente la hemos definido restando 
del año del accidente el año de nacimiento. Esta información es procesada para el conjunto del país por la Subdirección General de Estadísticas Sociales y Laborales del Ministerio de Trabajo y Asuntos Sociales ${ }^{17}$.

Los años estudiados han sido 1989, primer año de estadísticas consolidadas; 1993, cuando se produjo la incidencia más baja de todo el periodo; y 2000, por ser el último año disponible en el momento de realizar el estudio. Los datos analizados corresponden a la declaración individualizada de cada lesión por cualquier accidente de trabajo que produce baja laboral, pero ocurrido durante la jornada de trabajo, dejando fuera del análisis las lesiones por accidentes in-itinere (aquellos ocurridos en el desplazamiento del domicilio al trabajo o viceversa) y las recaídas. Igualmente, se han excluido las lesiones ocurridas en Ceuta y Melilla y en Organismos extraterritoriales. El total de LAT incluidas en este estudio fueron 594.240 no mortales y 1.277 mortales en $1989 ; 504.895$ no mortales y 985 mortales en 1993; y 898.562 no mortales y 1.904 mortales en 2000 .

Para calcular la incidencia bruta y específica para cada categoría de las variables analizadas, el denominador se ha obtenido a partir de los datos que proporciona la Encuesta de Población Activa ${ }^{18}$ para la población ocupada asalariada, en el segundo trimestre del año correspondiente.

La incidencia se ha calculado tanto para las LAT mortales como para las no mortales. La comparación entre CCAA se ha realizado después de ajustar la incidencia separadamente con las siguientes variables: sexo, edad (menos de 20, 20-24, 25-34, 35-44, 4554 y más de 54 años), actividad económica (dieciséis secciones de la Clasificación Nacional de Actividades Económicas) y tipo de contrato (indefinido y temporal), utilizando para ello un modelo de regresión de Poisson, que tuvo en cuenta la sobredispersión. Aquellas variables que introducían una variación superior al 10\%, cuando se compa- raban el riesgo relativo bruto y ajustado ${ }^{19}$ para la mayoría de las CCAA, son las se han tenido en cuenta en el modelo final. La representación gráfica se ha realizado clasificando las CCAA en terciles según el valor del riesgo relativo ajustado por actividad económica y tipo de contrato, que toma como valor de referencia la media española.

\section{RESULTADOS}

La incidencia de LAT no mortales fue alrededor de tres veces superior en hombres que en mujeres, observándose un número muy reducido de LAT mortales en las mujeres (tabla 1). Por otro lado, a diferencia de la incidencia de las LAT no mortales, la incidencia de LAT en los hombres se ha ido reduciendo durante los años estudiados: de 20,8 por 100.000 trabajadores en 1989 , a 16,7 en 1993 y 14,1 en 2000. En las mujeres, su tendencia es muy estable y muy inferior a los hombres.

La distribución de la incidencia por grupos de edad fue muy diferente para las LAT no mortales y las mortales (tabla 1). La incidencia de las LAT no mortales tiende a descender desde los grupos más jóvenes (147,6 por 1.000 trabajadores en 2000), en los que es más elevada, hasta los mayores de 45 a 54 años, en los que fue menor (59,7 en 2000). Este comportamiento se repite en los tres años analizados, aunque con valores superiores en casi todos los grupos de edad en el año 2000. Por el contrario, las LAT mortales siguen una distribución inversa a la anterior, incrementándose desde los grupos más jóvenes, en los que fue menor (4,6 por 100.000 trabajadores en 2000 en el de menos de 20 años y 5,1 en el de 20 a 24), hasta el grupo de mayor edad, en los que es más elevada (19,5 en 2000). Esto se repite en los tres años analizados, aunque con valores inferiores en casi todos los grupos de edad en el año 2000.

La incidencia de LAT no mortales fue entre dos y tres veces superior para los traba- 
Tabla 1

Distribución por sexo, edad y tipo de contratos de la frecuencia e incidencia de las lesiones mortales y no mortales con baja por accidente de trabajo en jornada. España, 1989, 1993 y 2000

\begin{tabular}{|c|c|c|c|c|c|c|}
\hline \multirow[b]{2}{*}{ Variables } & \multicolumn{2}{|c|}{1989} & \multicolumn{2}{|c|}{1993} & \multicolumn{2}{|c|}{2000} \\
\hline & Número & Incidencia & Número & Incidencia & Número & Incidencia \\
\hline & \multicolumn{6}{|c|}{ No mortales $(*)$} \\
\hline \multicolumn{7}{|l|}{ Sexo } \\
\hline Hombres & 539.217 & 89,1 & 438.191 & 76,7 & 741.937 & 98,5 \\
\hline mujeres & 55.023 & 20,4 & 66.704 & 22,6 & 156.625 & 33,6 \\
\hline \multicolumn{7}{|l|}{ Edad } \\
\hline$<20$ & 39.946 & 87,4 & 25.956 & 56,9 & 48.348 & 147,6 \\
\hline 20 a 24 & 101.236 & 84,9 & 74.535 & 82,8 & 159.557 & 118,0 \\
\hline 25 a 34 & 176.901 & 68,2 & 156.882 & 60,8 & 289.164 & 72,3 \\
\hline 35 a 44 & 130.209 & 65,3 & 117.630 & 52,4 & 207.893 & 63,1 \\
\hline 45 a 54 & 96.024 & 63,4 & 86.458 & 56,5 & 133.160 & 59,7 \\
\hline$>54$ & 49.924 & 49,8 & 43.434 & 45,4 & 60.440 & 61,2 \\
\hline \multicolumn{7}{|l|}{ Contrato } \\
\hline Indefinido & 290.474 & 45,2 & 241.064 & 41,0 & 367.310 & 44,4 \\
\hline Temporal & 303.766 & 130,6 & 263.831 & 94,5 & 531.252 & 135,6 \\
\hline \multirow[t]{2}{*}{ Total } & 594.240 & 67,9 & 504.895 & 58,2 & 898.562 & 73,7 \\
\hline & \multicolumn{6}{|c|}{ Mortales (†) } \\
\hline \multicolumn{7}{|l|}{ Sexo } \\
\hline Hombres & 1.258 & 20,8 & 954 & 16,7 & 1.063 & 14,1 \\
\hline mujeres & 19 & 0,7 & 31 & 1,0 & 31 & 0,7 \\
\hline \multicolumn{7}{|l|}{ Edad } \\
\hline$<20$ & 32 & 7,0 & 12 & 2,6 & 15 & 4,6 \\
\hline 20 a 24 & 135 & 11,3 & 70 & 7,8 & 69 & 5,1 \\
\hline 25 a 34 & 298 & 11,5 & 209 & 8,1 & 225 & 5,6 \\
\hline 35 a 44 & 272 & 13,6 & 241 & 10,7 & 297 & 9,0 \\
\hline 45 a 54 & 290 & 19,2 & 271 & 17,7 & 295 & 13,2 \\
\hline$>54$ & 250 & 24,9 & 182 & 19,0 & 193 & 19,5 \\
\hline \multicolumn{7}{|l|}{ Contrato } \\
\hline Indefinido & 705 & 11,0 & 570 & 9,7 & 560 & 6,8 \\
\hline Temporal & 572 & 24,6 & 415 & 14,9 & 534 & 13,6 \\
\hline Total & 1.277 & 14,6 & 985 & 11,4 & 1.094 & 9,0 \\
\hline
\end{tabular}

(*) Incidencia por 1.000; (†) Incidencia por 100.000 .

jadores con contratos temporales respecto a los trabajadores con contrato de trabajo indefinido (tabla 1). Para las LAT mortales la diferencia fue también importante, alrededor de dos veces superior en los trabajadores con contrato temporal. Tanto en las LAT no mortales como mortales, esta diferencia fue inferior en 1993.

Según la actividad económica de la empresa, observamos en la tabla 2 que la industria extractiva (228,8 por 1.000 trabajadores en 2000) y la construcción (173,3 en 2000) fueron en las que se observaron las incidencias de LAT no mortales más elevadas. Aunque también hay que destacar la pesca (119,2 en 2000), la industria manufacturera $(97,7$ en 2000) y la agricultura $(89,4$ en 2000). Por el contrario, donde hubo una menor incidencia fue en las empresas de intermediación financiara (5,2 en 2000) y de educación e investigación (6,6 en 2000). En relación a las LAT mortales (tabla 2), destaca también por su elevada incidencia la pesca ( 111,6 por 1.000 trabajadores), las industrias extractivas (44,5 en 2000), el transporte (27,9 en 2000) y la construcción (21,6 en 2000). Un patrón que se repite en los tres años estudiados.

Al comparar la incidencia de LAT no mortales (figura 3) por CCAA, ajustada por acti- 
Tabla 2

Incidencia de lesiones mortales y no mortales con baja por accidente de trabajo en jornada según actividad económica. España 1989, 1993 y 2000

\begin{tabular}{|c|c|c|c|c|c|c|}
\hline \multirow[b]{2}{*}{ Actividad económica (código CNAE-93) } & \multicolumn{3}{|c|}{ No mortales $(*)$} & \multicolumn{3}{|c|}{ Mortales ( $\dagger$ ) } \\
\hline & 1989 & 1993 & 2000 & 1989 & 1993 & 2000 \\
\hline Agricultura, ganadería, caza y selvicultura $(1,2)$ & 68,9 & 70,6 & 89,4 & 13,3 & 10,9 & 12,8 \\
\hline Pesca (5) & 78,4 & 87,4 & 119,2 & 99,9 & 60,2 & 111,6 \\
\hline Industrias extractivas (10 a 14) & 268,9 & 228,4 & 228,8 & 82,5 & 49,4 & 44,5 \\
\hline Industria manufacturera ( 15 a 37 ) & 110,9 & 87,6 & 97,7 & 14,1 & 11,6 & 7,8 \\
\hline $\begin{array}{l}\text { Producción y distribución de energía eléctrica, gas } \\
\text { y agua }(40,41)\end{array}$ & 36,1 & 35,2 & 35,4 & 18,5 & 20,4 & 9,3 \\
\hline Construcción (45) & 132,4 & 120,2 & 173,3 & 34,3 & 27,4 & 21,6 \\
\hline $\begin{array}{l}\text { Comercio; reparación de vehículos de motor, } \\
\text { motocicletas y ciclomotores y artículos } \\
\text { personales y de uso doméstico (50 a 52) }\end{array}$ & 59,6 & 58,7 & 70,1 & 13,3 & 9,3 & 5,7 \\
\hline Hostelería (55) & 59,9 & 64,4 & 76,6 & 6,6 & 4,1 & 2,8 \\
\hline $\begin{array}{l}\text { Transporte, almacenamiento y comunicaciones } \\
(60 \text { a 64) }\end{array}$ & 59,4 & 57,6 & 64,0 & 34,2 & 33,8 & 27,9 \\
\hline Intermediación financiera (65) & 5,8 & 5,7 & 5,2 & 4,1 & 3,8 & 1,6 \\
\hline $\begin{array}{l}\text { Actividades inmobiliarias y de alquiler; servicios } \\
\text { empresariales }(70 \text { a } 72,74)\end{array}$ & 22,9 & 17,3 & 78,3 & 12,3 & 5,2 & 6,3 \\
\hline $\begin{array}{l}\text { Administración pública, defensa y seguridad social } \\
\text { obligatoria (75) }\end{array}$ & 13,8 & 19,6 & 31,0 & 2,9 & 4,2 & 4,3 \\
\hline Educación e investigación $(73,80)$ & 4,5 & 6,5 & 6,6 & 0,9 & 1,0 & 0,7 \\
\hline $\begin{array}{l}\text { Actividades sanitarias y veterinarias, servicio } \\
\text { social ( } 85)\end{array}$ & 16,8 & 20,7 & 26,3 & 3,0 & 1,7 & 2,9 \\
\hline $\begin{array}{l}\text { Otras actividades sociales y de servicios prestados } \\
\text { a la comunidad; servicios personales (90 a 93) }\end{array}$ & 55,0 & 77,8 & 40,2 & 4,7 & 10,6 & 5,0 \\
\hline Hogares que emplean personal doméstico (95) & 1,6 & 2,9 & 2,5 & 3,5 & 1,2 & 1,5 \\
\hline Total & 67,9 & 58,2 & 73,7 & 14,6 & 11,4 & 9,0 \\
\hline
\end{tabular}

(*) Incidencia por 1.000; (†) Incidencia por 100.000 .

vidad económica y tipo de contrato, se observa un patrón en el que destaca la elevada incidencia en las Islas Baleares y Cataluña respecto al conjunto de España, siempre en el tercil superior en los tres años analizados. A las que se añade la Comunidad Valenciana, Asturias, País Vasco y Navarra en dos de los tres años analizados. Por el contrario, Galicia, Extremadura, Castilla-León y Aragón presentan una baja incidencia de estas lesiones, encontrándose en los tres años en el tercil inferior.

En relación a las lesiones mortales (figura 3 ), se observó una mayor incidencia en Asturias y el País Vasco en los tres años estudiados, aunque Galicia y Navarra también presenta una incidencia elevada durante dos de los tres años analizados. La incidencia más baja se encontró en Madrid y Cataluña en los tres años analizados.

\section{DISCUSIÓN}

Este estudio compara por primera vez en España la incidencia de LAT, mortales y no mortales entre CCAA, ajustando por diversas variables en tres años desde que esta información está disponible. Este es un problema apenas estudiado, si exceptuamos el reciente informe sobre riesgos laborales ${ }^{14}$, en el que se compara la incidencia bruta sin ajustar, y un trabajo preliminar de Castejón, 
Figura 1

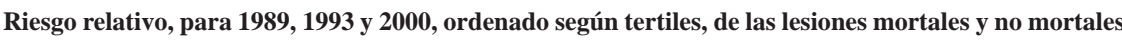
con baja por accidente de trabajo en jornada, ajustado por actividad económica y tipo de contrato en las Comunidades Autónomas, tomando como referencia la media española

Lesiones no mortales:

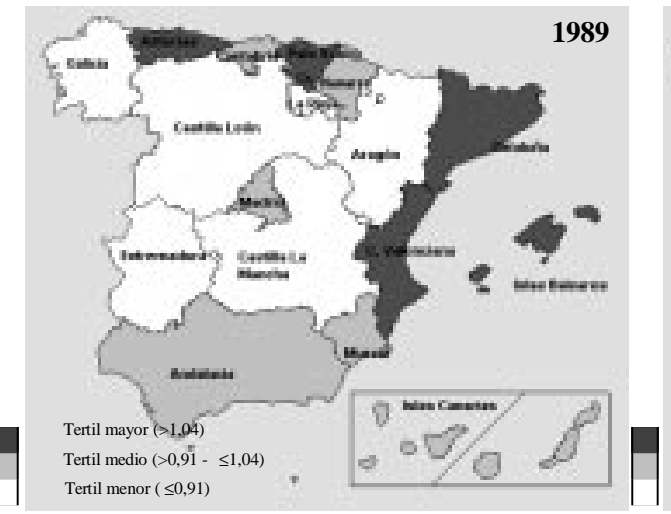

Lesiones mortales:

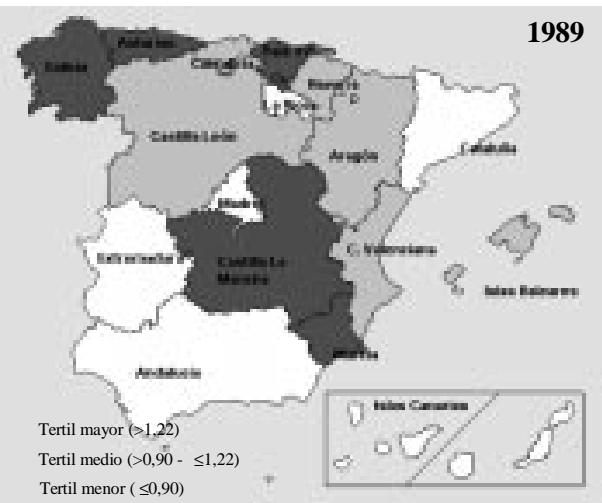

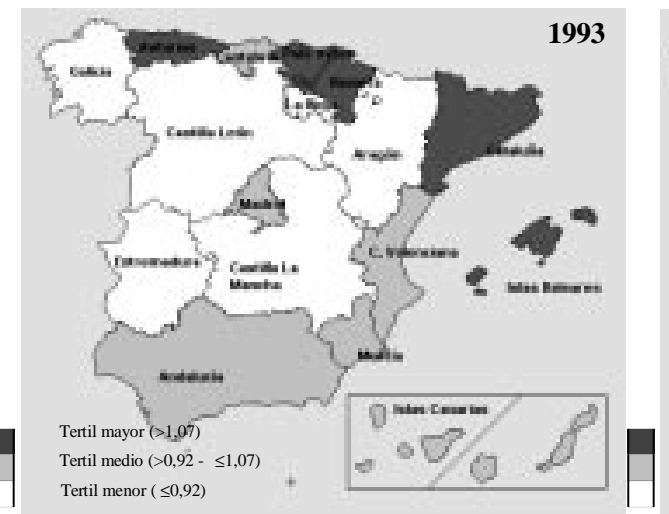
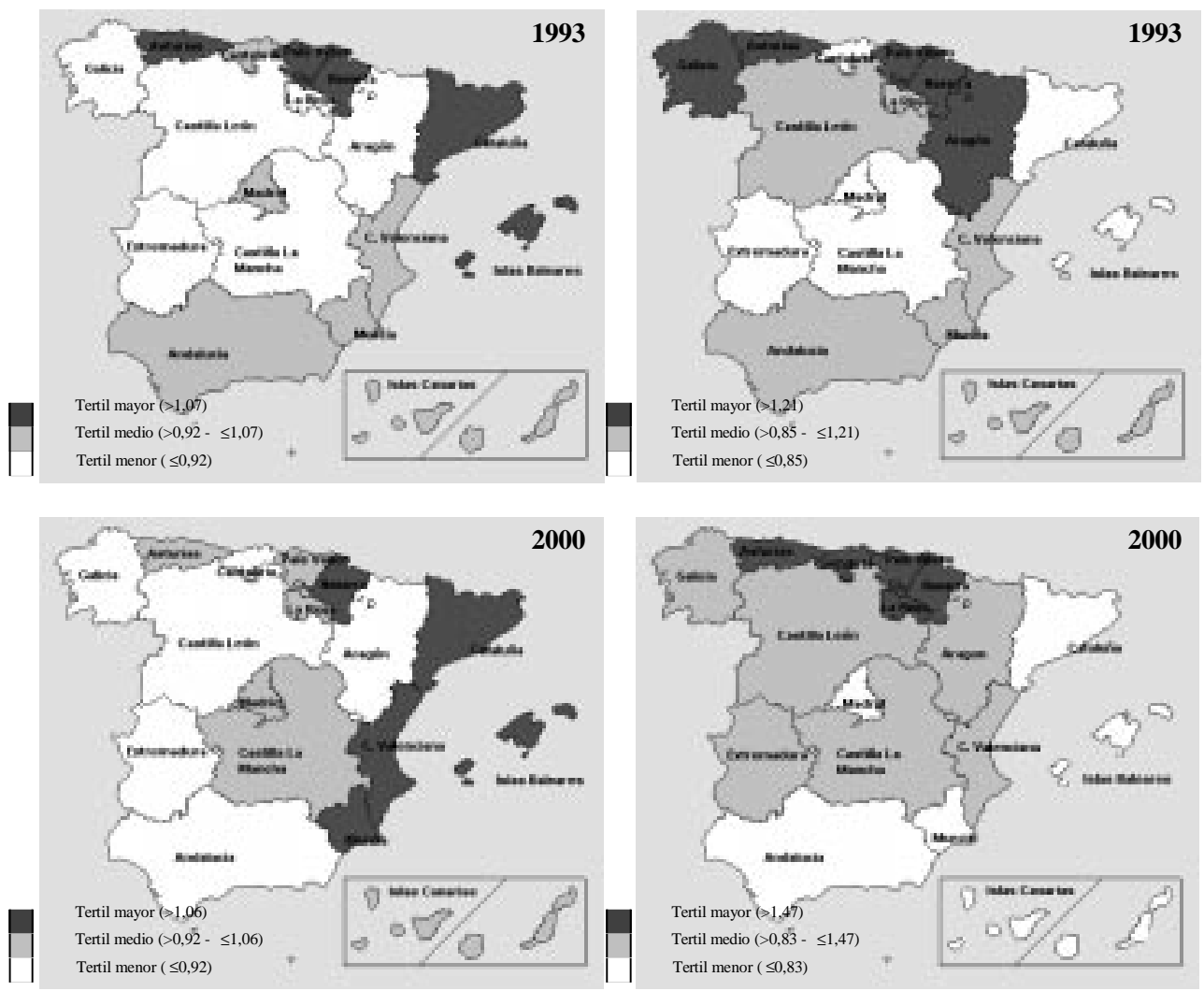
donde se estandariza únicamente por sector de actividad económica la incidencia para el total de lesiones ${ }^{20}$.

Los resultados de este estudio, que confirman los hallazgos preliminares, muestran que el riesgo de sufrir una LAT no se distribuye homogéneamente entre las CCAA. Igualmente, estos resultados permiten afirmar que hay CCAA que presentan una incidencia de LAT superior a la media española, que no estaría explicada ni por las diferencias según el tipo de contrato ni por las diferencias en la actividad económica de las empresas entre CCAA. Si bien, respecto a esta segunda variable, la heterogeneidad de actividades dentro de algunos grupos de actividades económicas utilizados en el estudio (como por ejemplo, la industria extractiva y sobre todo la manufacturera) y su localización preferente en determinadas CCAA, puede que no elimine completamente el efecto confusor de la actividad económica en la comparación.

El sexo y la edad tampoco modifican los resultados obtenidos, aunque éstos no han sido mostrados. Estas diferencias fueron especialmente significativas, por su elevada incidencia de LAT mortales en algunas CCAA de la cornisa cantábrica, y en relación a las no mortales en otras de la cuenca mediterránea.

Ahora bien, antes de concluir que estas diferencias observadas puedan ser reales, habría que descartar explicaciones que podemos calificar de espurias. Esto es, que las diferencias se deban a artefactos estadísticos como pueden ser, en este caso, que el nivel de declaración de lesiones sea diferente entre las CCAA o que los denominadores utilizados, la población ocupada asalariada que proporciona la Encuesta de Población Activa, tenga también una cobertura diferente entre las CCAA. Respecto a la primera hipótesis, hemos de señalar que no existen, hasta donde conocemos, estudios sobre la exhaustividad de la notificación de las LAT por $\mathrm{CCAA}^{21}$. Si bien, dado que la fiabilidad de la notificación depende de las Mutuas, las cuales desarrollan su actividad en el conjunto del Estado, aunque con diferente implantación territorial, podemos esperar que la calidad de la notificación se distribuya aleatoriamente entre las CCAA. Así, pues, es poco probable que esta hipótesis explique el exceso de lesiones mortales en Galicia y País Vasco, y de lesiones no mortales en Cataluña y Baleares, respecto al conjunto de España.

El problema de los denominadores utilizados en el análisis de las LAT es más difícil de descartar como explicación alternativa, pues es conocido que la población que proporciona la Encuesta de Población Activa no se corresponde exactamente con la población que tiene derecho a que se le reconozca una LAT a efectos económicos y sanitarios. Ello es especialmente importante en relación a los trabajadores autónomos agrícolas, del mar y de la minería del carbón, los cuales están concentrados en algunas CCAA. Sin embargo, un reciente estudio que estima para el año 2000 la incidencia de las LAT mortales y no mortales utilizando los datos de la Encuesta de Población Activa y los datos de afiliación de la Seguridad Social, considerando éstos como los más adecuados, encontró que el orden de magnitud de la incidencia en ambas series fue muy similar, con un coeficiente de Spearman de 0,9360 para la incidencia de lesiones no mortales y de 0,9525 para la incidencia de las morta$\mathrm{les}^{22}$. Por lo tanto, podemos deducir que es igualmente difícil que este problema de los denominadores explique las diferencias observadas.

Descartadas, provisionalmente, las explicaciones anteriores, y una vez que se ha ajustado por actividad económica y tipo de contrato, que son las variables más claramente relacionadas con la incidencia y con una distribución diferente por CCAA, la hipótesis más plausible a tener en cuenta sería que las empresas localizadas en las 
CCAA con mayor incidencia de lesiones presentan peores condiciones de trabajo. Esta hipótesis asume que las causas específicas de las lesiones están relacionadas con los equipos, los lugares de trabajo o las tareas que realizan los trabajadores; esto es, las condiciones de trabajo ${ }^{23}$. Los datos disponibles en el actual sistema de información de lesiones por accidentes de trabajo no permiten verificar esta hipótesis, y aunque estos puedan mejorar, tal como está previsto a partir del nuevo parte de accidente de traba$\mathrm{jo}^{24}$, siempre tiene una validez menor si este análisis se lleva a cabo con datos secundarios. La verificación de esta hipótesis requiere de datos primarios específicamente recogidos para este objetivo. Los estudios de casos y controles, donde el control es el mismo caso después de un tiempo relevante (case-crossover), parecen ser especialmente útiles en este tipo de fenómeno de aparición súbita, con un periodo de latencia muy cor$\operatorname{ta}^{25,26}$.

En relación a las LAT mortales hay que añadir una hipótesis auxiliar, relacionada con la influencia que sobre la letalidad pudieran tener los diferentes dispositivos asistenciales de las CCAA. En este sentido hay que decir que los recursos asistenciales para atender a las personas afectadas por LAT son gestionados por las Mutuas de Accidentes de Trabajo y Enfermedades Profesionales, aunque como es de esperar las atenciones urgentes se pueden realizar en cualquier servicio asistencial del Sistema Nacional de Salud.

La afirmación que las LAT son prevenibles, no sólo es teórica sino también, tal como afirmaba una reciente editorial del British Medical Journal ${ }^{27}$, algo contrastable empíricamente, como demuestran los países que están consiguiendo reducir las cifras de lesiones, principalmente mortales ${ }^{28}$. Ello será posible en España si somos capaces de desarrollar programas preventivos basados en la identificación previa de las causas.

\section{BIBLIOGRAFÍA}

1. Eurostat. European social statistics - Accidents at work and work-related health problems. Data 1994-2000. European Commission; 2003.

2. Dirección General de Ordenación Económica de la seguridad Social. Memoria económica-financiera y de gestión. Mutuas de Accidentes de Trabajo y Enfermedades Profesionales de la Seguridad Social. Ejercicio 2000. Madrid: Ministerio de Trabajo y Asuntos Sociales; 2002.

3. Veazie MA, Landen DD, Bender TR, Amandus HE. Epidemiologic research on the etiology of injuries at work. Annu Rev Public Health 1994; 15: 203-21.

4. Herrington TN, Morse LH. Occupational injuries. Evaluation, management and prevention. St Louis: Mosby Year Book Inc; 1995.

5. Salminen S, Kivimäki M, Elovainio M, Vahtera J. Stress factors predicting injuries of hospital personnel. Am J Ind Med 2003; 44: 32-6.

6. Sociedad Española de Epidemiología. Investigación. La investigación epidemiológica sobre condiciones de trabajo y salud en España. Madrid: INSHT; 1999.

7. Castejón E. Accidentalidad laboral en España. Salud y Trabajo 1992; 90: 4-11.

8. Boix P, Orts E, López MJ, Rodrigo F, Linares PJ. Modalidades de contratación y siniestralidad laboral en España en el periodo 1988-1995. Mapfre Seguridad 1998; 69: 15-27.

9. Villanueva V, Clemente I. Accidentes de trabajo y factores económicos asociados. Arch Prev Riesgos Labor 2001; 4: 6-15.

10. Castellá JL. Accidentes, empleo, carga de trabajo y peligrosidad del trabajo. Prevención, trabajo y salud 2000; 7 : 16-25.

11. Layana E, Abascal E, Artieda L et al. Determinantes de la accidentalidad laboral: condiciones versus relaciones de trabajo. Arch Prev Riesgos Labor 2003; 6: 120-4.

12. Benavides FG, Giráldez MT, Castejón E. Catot N, Zaplana M, Delclós J, Benach J, Gimeno D. Análisis de los mecanismos de producción de las lesiones leves por accidentes de trabajo en la construcción en España. Gac Sanit 2003; 17: 353-9.

13. Ley $31 / 1995$ de 8 de noviembre de Prevención de riesgos laborales. BOE núm. 269, de 10/11/1995.

Rev Esp Salud Pública 2004, Vol. 78, N. ${ }^{\circ} 5$ 
14. Durán F. Informe sobre los riesgos laborales y su prevención. Madrid: Presidencia del Gobierno; 2001 .

15. Plasència A, Cirera E. Accidentes de tráfico: un problema de salud a la espera de una respuesta sanitaria. Med Clín (Barc) 2003; 120: 378-9.

16. Orden de 16 de Diciembre de 1987; BOE del 29/12/1987.

17. Ministerio de Trabajo y Asuntos Sociales. (citado el 19 de septiembre de 2003). Disponible en: http:// www.mtas.es/insht/statistics/mtas.htm

18. Encuesta de Población Activa. Instituto Nacional de Estadística. (citado el 19 de septiembre de 2003). Disponible en: http://www.ine.es/epa02/ descripcion\% 20encuesta.pdf

19. Rothman K, Greenland S. Modern epidemiology ( $2^{\circ}$ edn.) Philadelphia: Lippincott-Raven; 1998: 256-7.

20. Castejón E. Estandarización de índices de accidentalidad. Libro de Resúmenes. XXV Congreso de Seguridad y Salud en el Trabajo. Valencia; 2001.

21. Benavides FG, Serra C. Evaluación de la calidad del sistema de información sobre las lesiones por accidentes de trabajo en España. Arch Prev Riesgos Labor 2003; 6: 26-30

22. Benavides FG, Catot N, Giráldez MT, Castejón E, Delclós J. Comparación de la incidencia de lesio- nes por accidente de trabajo según la Encuesta de Población Activa y el Registro de Afiliados a la Seguridad Social. Arch Prev Riesgos Labor 2004; 7(1): 16-21.

23. Benavides FG. Investigación y análisis estadístico de los accidentes de trabajo y enfermedades profesionales. En: Gestal JJ (ed.) Riesgos laborales en personal sanitario. Madrid: McGraw-Hill-Interamericana; 2003.p.103-15.

24. Orden TAS/2926/2002, de 19 de noviembre, por la que se establecen nuevos modelos para la notificación de los accidentes de trabajo. BOE núm. 279 de 21/11/2002.

25. Mittleman MA, Maldonado G, Gerberich SG, Smith GS, Sorock GS. Alternative approaches to analytical designs in occupational injury epidemiology. Am J Ind Med 1997; 32: 129-41.

26. Sorock GS, Lombardi DA, Hauser RB, Eisen EA, Herrick RF, Mittleman MA. A case-crossover study of occupational traumatic hand injury: methods and initial findings. Am J Ind Med 2001; 39: 171-9.

27. Davis RM, Pless B. BMJ bans «accidents». BMJ 2001; 322: 1320-1.

28. Loomis D, Bena JF, Bailer AJ. Diversity of trends in occupational injury mortality in the United States, 1980-96. Inj Prev 2003; 9: 9-14. 